\title{
SPECTRAL ASYMPTOTICS AND QUASICLASSICAL ANALYSIS OF SCHRÖDINGER TYPE OPERATORS*
}

\author{
ANDREA ZIGGIOTO ${ }^{\dagger}$
}

\begin{abstract}
In this work we consider a general class of Schrödinger type operators, associated to multi-quasi-elliptic symbols introduced by Buzano and Ziggioto in [9]. We develop their quasiclassical analysis and we obtain a uniform asymptotic formula for their counting function $\mathcal{N}_{\epsilon}(\tau)$, in the sense that it holds as $\tau \rightarrow+\infty$ and for all $0<\epsilon \leq 1$.
\end{abstract}

Key words. Spectral Theory, counting function, elliptic operators, quasiclassical analysis.

AMS subject classifications. 35P20, 47B06

1. Introduction. Quasiclassical analysis and spectral asymptotics are strictly related to each other (this is particularly evident when dealing with homogeneous symbols, see [6],Remark A.2.2). In both of them, the object of study is the counting function (which we denote by $\mathcal{N}(\tau)$ in the case of spectral asymptotics and by $\mathcal{N}_{\epsilon}(\tau)$ in the case of quasiclassical analysis) associated to the operators we are dealing with.

In spectral asymptotics we analyze the behavior of $\mathcal{N}(\tau)$ as $\tau \rightarrow+\infty$, while in quasiclassical analysis we study the behavior of $\mathcal{N}_{\epsilon}(\tau)$ as $\epsilon \rightarrow 0$, where $\epsilon$ plays the role of the Planck constant in Quantum Mechanics. ${ }^{(1)}$

In this paper we take into consideration multi-quasi-elliptic operators of Schrödinger type $h^{w}$, introduced by Buzano and Ziggioto in [9]. We already obtained an asymptotic formula for their counting function $\mathcal{N}(\tau)$ as $\tau \rightarrow+\infty$ and in particular we proved an estimate of the remainder term, showing that it always goes to 0 as $\tau \rightarrow+\infty$.

Now we consider quasiclassical operators associated to $h^{w}$ and their counting function $\mathcal{N}_{\epsilon}(\tau)$. Using the so called Tauberian condition (see condition 2. of Theorem 1 in Section 3), we manage to obtain a uniform asymptotic formula for $\mathcal{N}_{\epsilon}(\tau)$, in the sense that it is valid as $\tau \rightarrow+\infty$ and for all $0<\epsilon \leq 1$.

We can make a comparison with the results obtained in one of our previous papers, see [8]. In that case we treated quasiclassical analysis of more general operators (hypoelliptic operators), but we didn't manage to obtain a uniform asymptotic formula, holding as $\tau \rightarrow+\infty$ and for all $0<\epsilon \leq 1$. Moreover, in our uniform asymptotic formula obtained for multi-quasi-elliptic operators (see (10)) we don't need to exclude the critical values of the symbol $h(x, \xi)$ (i.e. the values $\tau$ for $\operatorname{which} \operatorname{grad} h(x, \xi)=0$ on the surface $\{(x, \xi): h(x, \xi)=\tau\})$.

We employ the following notation: given two functions $f, g: X \rightarrow \mathbb{R}$, and a subset $A \subset X$, we write

$$
f(x) \prec g(x), \quad \forall x \in A,
$$

if there exists a constant $C$ such that

$$
f(x) \leq C g(x), \quad \forall x \in A .
$$

\footnotetext{
*Received February 9, 2010; accepted for publication June 30, 2010.

${ }^{\dagger}$ Dipartimento di Matematica, Università di Torino, Via Carlo Alberto 10, 10123 Torino, Italia (andrea.ziggioto@unito.it).

${ }^{(1)}$ In order to be consistent with the notations used in [10] here we denote the Planck constant by $\epsilon$ and not by $h$, since we use $h$ to denote our operators.
} 
I would like to acknowledge Professor Buzano for his precious suggestions while writing this paper.

2. Multi-quasi-elliptic operators of Schrödinger type. We begin by recalling some basic notations and results about multi-quasi-elliptic weights and symbols. For references see [9], [1].

A convex polyhedron $\mathcal{P} \subset \mathbb{R}^{n}$ is the convex hull of a finite set of points in $\mathbb{R}^{n}$.

One can show that $\mathcal{P}$ can be obtained as the convex hull of a finite subset $V(\mathcal{P}) \subset$ $\mathbb{R}^{n}$ of points, which are convex linearly independent, called the vertices of $\mathcal{P}$ and univoquely determined by $\mathcal{P}$. Moreover if $(0,0, \ldots, 0) \in \mathcal{P}$, then there exists a finite set $N(\mathcal{P})=N_{0}(\mathcal{P}) \cup N_{1}(\mathcal{P}) \subset \mathbb{R}^{n}$ such that ${ }^{(2)}$

$$
\begin{aligned}
\mathcal{P}=\{x & \left.\in \mathbb{R}^{n} \mid \nu \cdot x \geq 0, \forall \nu \in N_{0}(\mathcal{P})\right\} \cap \\
& \cap\left\{x \in \mathbb{R}^{n} \mid \nu \cdot x \leq 1, \forall \nu \in N_{1}(\mathcal{P})\right\} .
\end{aligned}
$$

The boundary of $\mathcal{P}$ is made of faces $F_{\nu}$ which are the convex hull of the vertices of $\mathcal{P}$ lying on the hyperplane $H_{\nu}$ orthogonal to $\nu \in N(\mathcal{P})$ and of equation

$$
\begin{array}{ll}
\nu \cdot x=0, & \text { if } \nu \in N_{0}(\mathcal{P}), \\
\nu \cdot x=1, & \text { if } \nu \in N_{1}(\mathcal{P}) .
\end{array}
$$

We set

$$
F(\mathcal{P})=\bigcup_{\nu \in N_{1}(\mathcal{P})} F_{\nu}
$$

Definition 1. A complete polyhedron is a convex polyhedron $\mathcal{P} \subset \overline{\mathbb{R}_{+}^{n}}{ }^{\text {(3) }}$ with the following properties:

1. $V(\mathcal{P}) \subset \overline{\mathbb{R}_{+}^{n}}$;

2. $(0, \ldots, 0) \in V(\mathcal{P})$;

3. $V(\mathcal{P}) \neq\{(0, \ldots, 0)\}$;

4. $N_{0}(\mathcal{P})=\left\{\mathrm{e}_{1}, \ldots, \mathrm{e}_{n}\right\}$, with $\mathrm{e}_{j}=(0, \ldots, 0, \underset{j-\text { entry }}{1}, 0, \ldots, 0)$, for $j=1, \ldots, n$;

5. $N_{1}(\mathcal{P}) \subset \mathbb{R}_{+}^{n}$.

Consider now a complete polyhedron $\mathcal{P}$ with integer vertices:

$$
V(\mathcal{P}) \subset \mathbb{N}^{n} .
$$

To such a polyhedron we associate the multi-quasi-elliptic weight function:

$$
\Lambda(\xi ; \mathcal{P})=\left(\sum_{\alpha \in V(\mathcal{P}) \backslash 0} \xi^{2 \alpha}\right)^{1 / 2}
$$

Definition 2. Given a complete polyhedron $\mathcal{P}$, we set

$$
m(\mathcal{P})=\sup _{\nu \in N_{1}(\mathcal{P})} \max \left\{\frac{1}{\nu_{j}} \mid j=1, \ldots, n\right\} .
$$

(2) In $\mathbb{R}^{n}$ we always consider the norm $|x|=\left|x_{1}\right|+\cdots+\left|x_{n}\right|$.

(3) $\mathbb{R}_{+}=\{x \in \mathbb{R} \mid x>0\}$. 
$m(\mathcal{P})$ is called the formal order of $\mathcal{P}$.

DEFINITION 3. A multi-quasi-elliptic operator of Schrödinger type is a differential operator $h^{w}$ of domain $\mathcal{C}_{0}^{\infty}\left(\mathbb{R}^{n}\right)$ and Weyl symbol

$$
h(x, \xi)=p(x, \xi)+q(x)=\sum_{\alpha \in A} a_{\alpha}(x) \xi^{\alpha}+q(x)
$$

satisfying the following hypotheses.

1. The convex hull of $A$ is a complete polyhedron $\mathcal{P}$.

2. The potential $q$ is real valued and

$$
q(x) \geq 1, \quad \text { for all } x \in \mathbb{R}^{n} .
$$

3. There exists $0 \leq \delta<1 / m(\mathcal{P})$ such that for all $\beta \in \mathbb{N}^{n}$ we have

$$
\left|D^{\beta} q(x)\right| \prec q(x)^{1+\delta|\beta|}, \quad \forall x \in \mathbb{R}^{n} .
$$

4. The coefficients $a_{\alpha}$ are real valued.

5. There exists $0 \leq \rho<1$, such that for all $\alpha \in A$ and $\beta \in \mathbb{N}^{n}$, we have

$$
\left|D^{\beta} a_{\alpha}(x)\right| \prec q(x)^{(1-k(\alpha ; \mathcal{P})) \rho+\delta|\beta|} \quad \forall x \in \mathbb{R}^{n},
$$

where

$$
k(\alpha ; \mathcal{P})=\inf \left\{t>0 \mid t^{-1} \alpha \in \mathcal{P}\right\}=\max _{\nu \in N_{1}(\mathcal{P})} \nu \cdot \alpha .
$$

6. There exists $R_{0} \geq 0$ such that

$$
p_{0}(x, \xi) \succ \Lambda(\xi ; \mathcal{P}), \quad \forall|\xi| \geq R_{0}, \forall x \in \mathbb{R}^{n},
$$

where

$$
p_{0}(x, \xi)=\sum_{\alpha \in A \cap F(\mathcal{P})} a_{\alpha}(x) \xi^{\alpha}
$$

is the principal symbol of $p^{w}$.

REMARK. We can say that multi-quasi-elliptic symbols generalize elliptic and quasi-elliptic symbols. More specifically, limiting ourselves to dimension $n=2$, we can represent the complete polyhedron $\mathcal{P}$ associated to a multi-quasi-elliptic symbol as a polygon with more than one face, as it is shown in Figure 1. The complete polyhedron $\mathcal{P}$ associated to an elliptic symbol can be represented instead as an isosceles triangle, as shown in Figure 2. Finally, the complete polyhedron $\mathcal{P}$ associated to a quasi-elliptic symbol can be represented as a right-angled triangle, as shown in Figure 3.

We are going to use the Weyl-Hörmander calculus with locally temperate metrics and weights: see [2], and [4] for more details.

Let

$$
\lambda(x, \xi)=\left\{\Lambda(\xi ; \mathcal{P})^{2}+q(x)^{2}\right\}^{1 / 2} .
$$

Then the Riemannian metric

$$
g_{x, \xi}(y, \eta)=\lambda(x, \xi)^{2 \delta}|y|^{2}+\lambda(x, \xi)^{-2 / m(\mathcal{P})}|\eta|^{2}
$$




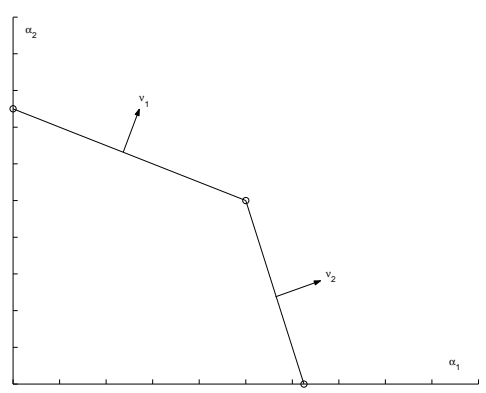

FIG. 1. multi-quasi-elliptic case $\xi_{1}^{12}+\xi_{1}^{10} \xi_{2}^{10}+\xi_{2}^{14}$

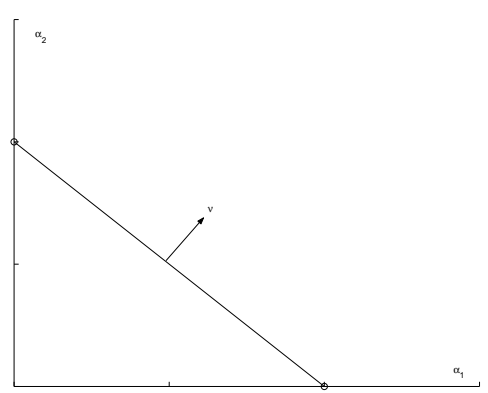

FIG. 2. elliptic case $\xi_{1}^{2}+\xi_{2}^{2}$

(where $\delta$ is the same as in (1)) is locally $\sigma$ temperate with respect to the slowly varying metric

$$
G_{x}(t)=|t|^{2} q(x)^{2 \delta}
$$

In particular $\lambda$ is locally $\sigma, g$ temperate with respect to $G$. See [9], Proposition 3, for further details. Moreover we can show the following result:

Proposition 1. The so called principle of indetermination is satisfied by the metric $g$ defined in (4), that is

$$
\sup _{x, \xi} \frac{g_{x, \xi}(y, \eta)}{g_{x, \xi}^{\sigma}(y, \eta)}<+\infty
$$

Proof. In the case of the metric $g$ defined in (4) it is standard to show that

$$
\sup _{x, \xi} \frac{g_{x, \xi}}{g_{x, \xi}^{\sigma}}=\lambda(x, \xi)^{2\left(\delta-\frac{1}{m(\mathcal{P})}\right)} .
$$

Then, since $0 \leq \delta<\frac{1}{m(\mathcal{P})}$ and $q(x) \geq 1$ for all $x \in \mathbb{R}^{n}$, we have that

$$
\sup _{x, \xi} \frac{g_{x, \xi}(y, \eta)}{g_{x, \xi}^{\sigma}(y, \eta)} \leq 1
$$

Finally, we define the counting function associated to the operator $h^{w}$ :

$$
\mathcal{N}_{h}(\tau)=\text { number of eigenfunctions of the closure of } h^{w} \text { corre- }
$$
sponding to eigenvalues less or equal to $\tau$. 


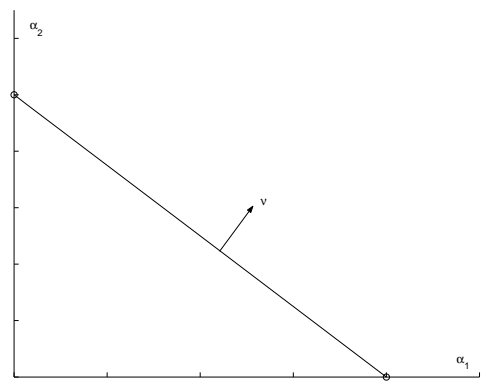

FIG. 3. quasi-elliptic case $\xi_{1}^{4}+\xi_{2}^{6}$

3. Quasiclassical Analysis of Multi-Quasi-Elliptic Operators of Schrödinger Type. Consider a multi-quasi-elliptic operator of Schrödinger type $h^{w}$.

Let us introduce the operator $h_{\epsilon}^{w}$ whose Weyl symbol is

$$
h_{\epsilon}(x, \xi)=h(\epsilon x, \epsilon \xi)=p(\epsilon x, \epsilon \xi)+q(\epsilon x),
$$

where $\epsilon$ is a real parameter such that $0<\epsilon \leq 1$.

Starting from the metrics defined in (4), let us define the following new Riemannian metrics ${ }^{\epsilon} g_{x, \xi}(y, \eta)$ and ${ }^{\epsilon} G_{x}(t)$ in this way:

$$
{ }^{\epsilon} g_{x, \xi}(y, \eta)=g_{\epsilon x, \epsilon \xi}(\epsilon y, \epsilon \eta)=\epsilon^{2}\left(\lambda(\epsilon x, \epsilon \xi)^{2 \delta}|y|^{2}+\lambda(\epsilon x, \epsilon \xi)^{-\frac{2}{m(\mathcal{P})}}|\eta|^{2}\right)
$$

and

$$
{ }^{\epsilon} G_{x}(t)=G_{\epsilon x}(\epsilon t)=\epsilon^{2}|t|^{2} q(\epsilon x)^{2 \delta} .
$$

We know that $g_{x, \xi}(y, \eta)$ is locally $\sigma$ temperate with respect to $G_{x}(t)$ and that $\lambda$ is locally $\sigma, g$ temperate with respect to $G_{x}(t)$. Therefore, it follows that also ${ }^{\epsilon} g_{x, \xi}$ is slowly varying, locally $\sigma$ temperate with respect to ${ }^{\epsilon} G_{x}(t)$ for all $0<\epsilon \leq 1$ and that also $\lambda(\epsilon x, \epsilon \xi)$ is locally $\sigma, g$ temperate with respect to ${ }^{\epsilon} G_{x}(t)$, for all $0<\epsilon \leq 1$ (see $[3],[8])$.

Let us analyze the principle of indetermination in the case of the metric ${ }^{\epsilon} g$.

Proposition 2. We have that the principle of indetermination is satisfied by the new metric ${ }^{\epsilon} g$, for all $0<\epsilon \leq 1$.

Proof. We have

$$
\begin{aligned}
\sup _{x, \xi} \frac{{ }^{\epsilon} g_{x, \xi}}{\left({ }^{\epsilon} g\right)_{x, \xi}^{\sigma}} & =\epsilon^{4} \lambda(\epsilon x, \epsilon \xi)^{2\left(\delta-\frac{1}{m(\mathcal{P})}\right)}= \\
& =\left(\lambda(\epsilon x, \epsilon \xi) \epsilon^{\frac{2 m(\mathcal{P})}{\delta m(\mathcal{P})-1}}\right)^{2\left(\delta-\frac{1}{m(\mathcal{P})}\right)}= \\
& =\lambda_{\epsilon}(x, \xi)^{2\left(\delta-\frac{1}{m(\mathcal{P})}\right)},
\end{aligned}
$$

for all $0<\epsilon \leq 1$. Therefore, repeating the same arguments of the proof of Proposition 1 , we obtain that the principle of indetermination is satisfied also by ${ }^{\epsilon} g$.

Due to this proposition, from now on we will work with the following symbol:

$$
H_{\epsilon}(x, \xi)=\epsilon^{\frac{2 m(\mathcal{P})}{\delta m(\mathcal{P})-1}} h_{\epsilon}(x, \xi) .
$$


Now we formulate Proposition 4 of [9] in this new context:

Proposition 3. Consider a multi-quasi-elliptic operator of Schrödinger type $h^{w}=p^{w}+q$. If $q(x) \rightarrow+\infty$ then the operator $H_{\epsilon}^{w}$, corresponding to the new symbol $H_{\epsilon}$, is semi-bounded from below and essentially self-adjoint in $L^{2}\left(\mathbb{R}^{n}\right)$, for all $0<\epsilon \leq$ 1.

Moreover its closure in $L^{2}\left(\mathbb{R}^{n}\right)$ has discrete spectrum diverging to $+\infty$.

Proof. Thanks to Proposition 2 of [9] (which is trivially satisfied also by $H_{\epsilon}$ and $\left.\lambda_{\epsilon}\right)$, there exists $c_{0}>0$ such that

$$
\lambda_{\epsilon}(x, \xi) \prec c_{0} \epsilon^{\frac{2 m(\mathcal{P})}{1-\delta m(\mathcal{P})}}+H_{\epsilon}(x, \xi) \prec \lambda_{\epsilon}(x, \xi), \quad \forall(x, \xi) \in \mathbb{R}^{n} \times \mathbb{R}^{n},
$$

for all $0<\epsilon \leq 1$. Then $\widetilde{H}_{\epsilon}=c_{0} \epsilon^{\frac{2 m(\mathcal{P})}{1-\delta m(\mathcal{P})}}+H_{\epsilon}$ is locally temperate for all $0<\epsilon \leq 1$. Thanks to Proposition 2 of [9] again, it is easy to check that $\widetilde{H}_{\epsilon}$ belongs to the class of Weyl-Hörmander $S\left(\widetilde{H}_{\epsilon},{ }^{\epsilon} g\right)$ for all $0<\epsilon \leq 1$ and the result is a special case of Proposition 6.1 of [2].

Remark. Thanks to Proposition 3 we can define the counting function of the closure of the operator $H_{\epsilon}^{w}$ :

$$
\begin{aligned}
\mathcal{N}_{H_{\epsilon}}(\tau)= & \text { number of eigenfunctions of the closure of } H_{\epsilon}^{w}, \text { cor- } \\
& \text { responding to eigenvalues less than or equal to } \tau .
\end{aligned}
$$

It is clear that Proposition 3 also applies to

$$
H_{0, \epsilon}=h_{0, \epsilon} \epsilon^{\frac{2 m(\mathcal{P})}{\delta m(\mathcal{P})-1}},
$$

where $h_{0, \epsilon}$ is the symbol of the principal part of $h_{\epsilon}^{w}$, that is

$$
h_{0, \epsilon}(x, \xi)=p_{0}(\epsilon x, \epsilon \xi)+q(\epsilon x) .
$$

In particular we have that $H_{0, \epsilon}^{w}$ is essentially self-adjoint and that its closure has a discrete spectrum diverging to $+\infty$ (see also Proposition 4 of [9]).

Before claiming our main result, we have to state the following theorem, which is a direct consequence of Proposition 5 of [9]:

Proposition 4. Consider a multi-quasi-elliptic operator of Schrödinger type $h^{w}$ and assume that $q(x) \rightarrow+\infty$ as $|x| \rightarrow+\infty$. If there exists $k>0$ such that

$$
h_{0}^{-k} \in L^{1}\left(\mathbb{R}^{2 n}\right)
$$

then there exists $\tau_{0}$ such that

$$
\mathcal{N}_{H_{0, \epsilon}}(\tau)=\mathcal{W}\left(\tau ; H_{0, \epsilon}\right)\left\{1+O\left(\mathcal{R}_{\epsilon, \mu_{0}}\right)\right\},
$$

for all $\tau \geq \tau_{0}$, uniformly with respect to $0<\epsilon \leq 1$, where

$$
\begin{gathered}
\mathcal{W}\left(\tau ; H_{0, \epsilon}\right)=(2 \pi)^{-n} \iint_{H_{0, \epsilon} \leq \tau} d x d \xi \\
\mathcal{R}_{\epsilon, \mu_{0}}(\tau)=\frac{\mathcal{W}\left(\tau+\tau^{1-\mu_{0}} ; H_{0, \epsilon}\right)-\mathcal{W}\left(\tau-\tau^{1-\mu_{0}} ; H_{0, \epsilon}\right)}{\mathcal{W}\left(\tau ; H_{0, \epsilon}\right)},
\end{gathered}
$$


and

$$
0<\mu_{0}<\frac{2}{3} \frac{1-\delta m(\mathcal{P})}{m(\mathcal{P})}
$$

Proof. By means of a change of coordinates we immediately obtain that

$$
\begin{aligned}
\left\|H_{0, \epsilon}^{-k}\right\|_{L^{1}} & =\epsilon^{\frac{2 k m(\mathcal{P})}{1-\delta m(\mathcal{P})}} \int\left|h_{0, \epsilon}(x, \xi)\right|^{-k} d x d \xi= \\
& =\epsilon^{\frac{2 k m(\mathcal{P})}{1-\delta m(\mathcal{P})}} \int\left|h_{0}(\epsilon x, \epsilon \xi)\right|^{-k} d x d \xi= \\
& =\epsilon^{\frac{2 k m(\mathcal{P})}{1-\delta m(\mathcal{P})}-2 n} \int\left|h_{0}(x, \xi)\right|^{-k} d x d \xi \leq \\
& \leq \int\left|h_{0}(x, \xi)\right|^{-k} d x d \xi=\left\|h_{0}^{-k}\right\|_{L^{1}},
\end{aligned}
$$

if we take $\frac{2 k m(\mathcal{P})}{1-\delta m(\mathcal{P})}-2 n \geq 0$, that is

$$
\frac{1}{m(\mathcal{P})}-\frac{k}{n} \leq \delta<\frac{1}{m(\mathcal{P})}
$$

Therefore we obtain that the integrability of $h_{0}^{-k}(x, \xi)$ implies the integrability of $H_{0, \epsilon}^{-k}(x, \xi)$ and that the $L^{1}$ norm of $H_{0, \epsilon}^{-k}$ is uniformly bounded with respect to $0<$ $\epsilon \leq 1$. The remaining part of the proof is an immediate consequence of Proposition 5 in $[9]$.

Now we can state and prove our main result.

THEOREM 1. Let $\mathcal{N}_{\epsilon}(\tau)$ be the counting function associated to the operator $h_{\epsilon}^{w}$. Assume that

1. $q(x) \rightarrow+\infty$, as $|x| \rightarrow+\infty$,

2. there exists $\tau_{0} \geq 0$, such that

$$
\mathcal{V}(2 \tau) \prec \mathcal{V}(\tau), \quad \forall \tau \geq \tau_{0},
$$

where

$$
\mathcal{V}(\tau)=\int_{q(x) \leq \tau} d x .
$$

3. for all $r>0$ we have

$$
\inf _{\substack{x \in \mathbb{R}^{n} \\|\xi| \geq r}} p_{0}(x, \xi)>0
$$

4. there exist $t_{0}>0, \omega \in \mathbb{R}_{+}^{n}$ and $C_{0}>0$, such that

$$
p_{0}\left(x,(1+t)^{\omega} \xi\right) \geq\left(1+C_{0} t\right) p_{0}(x, \xi),
$$

for all $0<t<t_{0}$ and all $(x, \xi) \in \mathbb{R}^{n} \times \mathbb{R}^{n}$, where

$$
(1+t)^{\omega} \xi=\left((1+t)^{\omega_{1}} \xi_{1}, \ldots,(1+t)^{\omega_{n}} \xi_{n}\right) .
$$


Then for

$$
\begin{gathered}
0<d<\frac{4}{3} \frac{|\nu|}{1+|\nu|} \\
0<\mu<\frac{2}{3} \frac{1-\delta m(\mathcal{P})}{m(\mathcal{P})} \frac{|\nu|}{1+|\nu|}, \\
\mu \leq(1-\rho)(1-\zeta) \frac{|\nu|}{1+|\nu|},
\end{gathered}
$$

with

$$
\frac{|\nu|}{1+|\nu|}=\max _{\tilde{\nu} \in N_{1}(\mathcal{P})} \frac{|\tilde{\nu}|}{1+|\tilde{\nu}|}
$$

and

$$
\zeta=\max _{\alpha \in A \backslash F(\mathcal{P})} k(\alpha ; \mathcal{P})
$$

we have that

$$
\mathcal{N}_{\epsilon}(\tau)=\epsilon^{-2 n} \mathcal{W}\left(\tau ; h_{0}\right)\left\{1+O\left(\epsilon^{d} \tau^{-\mu}\right)\right\},
$$

as $\tau \rightarrow+\infty$ and for all $0<\epsilon \leq 1$, where

$$
\mathcal{W}\left(\tau ; h_{0}\right)=(2 \pi)^{-n} \int_{h_{0} \leq \tau} d x d \xi .
$$

REMARK. As already remarked in the introduction, in order to obtain the result of Theorem 1, we don't need to avoid the critical values of the symbol $h(x, \xi)$ from our asymptotic formula, as instead we are compelled to do in the case of hypoelliptic operators (see [10]). Moreover, in the case of hypoelliptic operators we don't have a uniform asymptotic formula, in the sense that it holds only as $\epsilon \rightarrow 0$ (see [8]).

4. Proof of Theorem 1. In order to prove Theorem 1, let us begin to estimate the remainder term $\mathcal{R}_{\epsilon, \mu_{0}}(\tau)$ for the counting function associated to $h_{0, \epsilon}^{w}$, as $\tau \rightarrow+\infty$ and for all $0<\epsilon \leq 1$.

Proposition 5. Under the same hypotheses of Theorem 1 we have that

$$
\mathcal{N}_{h_{0, \epsilon}}(\tau)=\epsilon^{-2 n} \mathcal{W}\left(\tau ; h_{0}\right)\left\{1+O\left(\epsilon^{d} \tau^{-\mu}\right)\right\},
$$

as $\tau \rightarrow \infty$ and for all $0<\epsilon \leq 1$, where

$$
0<\mu<\frac{2}{3} \frac{1-\delta m(\mathcal{P})}{m(\mathcal{P})} \frac{|\nu|}{1+|\nu|}
$$

and

$$
0<d<\frac{4}{3} \frac{|\nu|}{1+|\nu|}
$$


Proof. Since $\mathcal{N}_{h_{0, \epsilon}}(\tau)$ is the counting function associated to the operator $h_{0, \epsilon}^{w}$, then it is clear that $H_{0, \epsilon}^{w}$ has exactly $\mathcal{N}_{h_{0, \epsilon}}\left(\epsilon^{\frac{2 m(\mathcal{P})}{1-\delta m(\mathcal{P})}} \tau\right)$ eigenvalues less than or equal to $\tau$ and that

$$
\mathcal{W}\left(\tau ; H_{0, \epsilon}\right)=(2 \pi)^{-n} \iint_{h_{0}(\epsilon x, \epsilon \xi) \epsilon^{\frac{2 m(\mathcal{P})}{\delta m(\mathcal{P})-1}} \leq \tau} d x d \xi=\epsilon^{-2 n} \mathcal{W}\left(\epsilon^{\frac{2 m(\mathcal{P})}{1-\delta m(\mathcal{P})}} \tau ; h_{0}\right) .
$$

Thanks to Proposition 4 , we obtain that for all $0<\mu_{0}<\frac{2}{3} \frac{1-\delta m(\mathcal{P})}{m(\mathcal{P})}$ there exists a real number $C_{\mu_{0}}>0$ such that

$$
\begin{aligned}
& \left|\mathcal{N}_{h_{0, \epsilon}}\left(\epsilon^{\frac{2 m(\mathcal{P})}{1-\delta m(\mathcal{P})}} \tau\right)-\epsilon^{-2 n} \mathcal{W}\left(\epsilon^{\frac{2 m(\mathcal{P})}{1-\delta m(\mathcal{P})}} \tau ; h_{0}\right)\right| \leq \\
& \quad \leq C_{\mu_{0}} \epsilon^{-2 n}\left(\mathcal{W}\left(\epsilon^{\frac{2 m(\mathcal{P})}{1-\delta(\mathcal{P})}}\left(\tau+\tau^{1-\mu_{0}}\right) ; h_{0}\right)-\mathcal{W}\left(\epsilon^{\frac{2 m(\mathcal{P})}{1-\delta m(\mathcal{P})}}\left(\tau-\tau^{1-\mu_{0}}\right) ; h_{0}\right)\right),
\end{aligned}
$$

as $\tau \rightarrow+\infty$, for all $0<\epsilon \leq 1$.

Letting $\epsilon^{\frac{2 m(\mathcal{P})}{1-\delta m(\mathcal{P})}} \tau=\lambda$ and provided that also $\lambda \epsilon^{\frac{2 m(\mathcal{P})}{\delta m(\mathcal{P})-1}}$ is sufficiently large, we obtain:

$$
\begin{aligned}
& \left|\mathcal{N}_{h_{0, \epsilon}}(\lambda)-\epsilon^{-2 n} \mathcal{W}\left(\lambda ; h_{0}\right)\right| \leq \\
& \quad \leq C_{\mu_{0}} \epsilon^{-2 n}\left(\mathcal{W}\left(\lambda\left(1+\epsilon^{\frac{2 m(\mathcal{P}) \mu_{0}}{1-\delta m(\mathcal{P})}} \lambda^{-\mu_{0}}\right) ; h_{0}\right)-\mathcal{W}\left(\lambda\left(1-\epsilon^{\frac{2 m(\mathcal{P}) \mu_{0}}{1-\delta m(\mathcal{P})}} \lambda^{-\mu_{0}} ; h_{0}\right)\right)\right)
\end{aligned}
$$

for $\lambda$ large enough, $0<\mu_{0}<\frac{2}{3} \frac{1-\delta m(\mathcal{P})}{m(\mathcal{P})}$ and $0<\epsilon \leq 1$.

Recalling Lemma 2 of [9] and letting $\theta=\epsilon^{\frac{2 m(\mathcal{P}) \mu_{0}}{1-\delta m(\mathcal{P})}} \lambda^{-\mu_{0}}$ we obtain that

$$
\begin{aligned}
\mathcal{W}\left(\lambda\left(1+\epsilon^{\frac{2 m(\mathcal{P}) \mu_{0}}{1-\delta m(\mathcal{P})}} \lambda^{-\mu_{0}}\right) ; h_{0}\right)- & \mathcal{W}\left(\lambda\left(1-\epsilon^{\frac{2 m(\mathcal{P}) \mu_{0}}{1-\delta m(\mathcal{P})}} \lambda^{-\mu_{0}}\right) ; h_{0}\right) \prec \\
& \prec \lambda^{-\mu_{0} \frac{|\nu|}{1+\nu}} \epsilon^{\frac{2 m(\mathcal{P}) \mu_{0}}{1-\delta m(\mathcal{P})} \frac{|\nu|}{1+\mid \nu}} \mathcal{W}\left(\lambda ; h_{0}\right),
\end{aligned}
$$

for all $\epsilon$ and $\lambda$ such that $\lambda \geq \tau_{1}$ and $0<\epsilon \leq \theta_{0}^{\frac{1-\delta m(\mathcal{P})}{2 m(\mathcal{P}) \mu_{0}}}$ (we can suppose $\tau_{1} \geq 1$ without any restriction). Therefore, if we let

$$
\begin{gathered}
\mu=\mu_{0} \frac{|\nu|}{1+|\nu|}, \\
d=\frac{2 m(\mathcal{P}) \mu_{0}}{1-\delta m(\mathcal{P})} \frac{|\nu|}{1+|\nu|},
\end{gathered}
$$

we have that formula (11) holds with

$$
\begin{aligned}
0<\mu & <\frac{2}{3} \frac{1-\delta m(\mathcal{P})}{m(\mathcal{P})} \frac{|\nu|}{1+|\nu|}, \\
0 & <d<\frac{4}{3} \frac{|\nu|}{1+|\nu|} .
\end{aligned}
$$

We still need the following result:

Proposition 6. Under the same hypotheses of Theorem 1, there exist $C_{2} \geq 1$ and $\tau_{2} \geq 0$ such that

$$
\mathcal{N}_{h_{0, \epsilon}}\left(\tau-C_{2} \tau^{1-(1-\zeta)(1-\rho)}\right) \leq \mathcal{N}_{\epsilon}(\tau) \leq \mathcal{N}_{h_{0, \epsilon}}\left(\tau+C_{2} \tau^{1-(1-\zeta)(1-\rho)}\right),
$$


for all $\tau \geq \tau_{2}$, for all $0<\epsilon \leq 1$.

Proof. The operators $h_{\epsilon}^{w}$ and $h_{0, \epsilon}^{w}$ have the same domain $D$, because they have the same principal symbol. Then we have the following variational characterizations of the counting function. If $\mathcal{L}$ is the set of all linear subspaces of $D$, then

$$
\mathcal{N}_{\epsilon}(\tau)=\inf \left\{\operatorname{codim} L \mid L \in \mathcal{L}:\left(h_{\epsilon}^{w} u, u\right)>\tau\|u\|_{L^{2}}, \forall u \in L\right\}
$$

and

$$
\mathcal{N}_{0, \epsilon}(\tau)=\inf \left\{\operatorname{codim} L \mid L \in \mathcal{L}:\left(h_{0, \epsilon}^{w} u, u\right)>\tau\|u\|_{L^{2}}, \forall u \in L\right\} .
$$

In the proof of Proposition 5.1 of [5], it is shown that (14) and (15) together with Proposition 7 in [9] imply (13) and the constant $C_{2}$ can be chosen uniformly with respect to $0<\epsilon \leq 1$.

Now we are ready to prove our main theorem.

Proof of Theorem 1. From (11) we have

$$
\begin{aligned}
\mathcal{N}_{h_{0, \epsilon}}(\tau & \left.+C_{2} \tau^{1-(1-\zeta)(1-\rho)}\right) \leq \\
& \leq \epsilon^{-2 n} \mathcal{W}\left(\tau+C_{2} \tau^{1-(1-\zeta)(1-\rho)} ; h_{0}\right)\left\{1+O\left(\epsilon^{d} \tau^{-\mu}\right)\right\},
\end{aligned}
$$

as $\tau \rightarrow+\infty$ and for all $0<\epsilon \leq 1$. From (42) in Lemma 2 of [9], we have

$$
\begin{aligned}
\mathcal{W}(\tau & \left.+C_{2} \tau^{1-(1-\zeta)(1-\rho)} ; h_{0}\right) \leq \\
& \leq \mathcal{W}\left(\tau ; h_{0}\right)\left\{1+O\left(\tau^{-(1-\zeta)(1-\rho) \frac{|\nu|}{1+|\nu|}}\right)\right\}
\end{aligned}
$$

and therefore

$$
\begin{aligned}
\mathcal{N}_{h_{0, \epsilon}}(\tau & \left.+C_{2} \tau^{1-(1-\zeta)(1-\rho)}\right) \leq \\
& \leq \epsilon^{-2 n} \mathcal{W}\left(\tau ; h_{0}\right)\left\{1+O\left(\epsilon^{d} \tau^{-\mu}\right)\right\}\left\{1+O\left(\tau^{-(1-\zeta)(1-\rho) \frac{|\nu|}{1+|\nu|}}\right)\right\} \\
& \leq \epsilon^{-2 n} \mathcal{W}\left(\tau ; h_{0}\right)\left\{1+O\left(\epsilon^{d} \tau^{-\mu}\right)\right\},
\end{aligned}
$$

with $d$ satisfying (7) and $\mu$ satisfying (8) and (9). Using (43) in Lemma 2 of [9], we obtain in the same way the other estimate:

$$
\mathcal{N}_{h_{0, \epsilon}}\left(\tau-C_{2} \tau^{1-(1-\zeta)(1-\rho)}\right) \geq \epsilon^{-2 n} \mathcal{W}\left(\tau ; h_{0}\right)\left\{1+O\left(\epsilon^{d} \tau^{-\mu}\right)\right\} .
$$

Formula (10) now follows from (16), (17) and (13). The proof of Theorem 1 is complete.

5. An example: the quasi-elliptic case. At the end of our paper we take into exam the special case in which $p_{0}(x, \xi)$ is quasi-elliptic with respect to $\xi$, that is

$$
p_{0}\left(x, t^{\omega} \xi\right)=t p_{0}(x, \xi) \quad \forall t \in \mathbb{R}_{+}, \forall x, \xi \in \mathbb{R}^{n},
$$

where $\omega \in \mathbb{R}_{+}^{n}$ is defined in assumption 4 of Theorem 1 . $6)$

In the quasi-elliptic case, (42) and (43) in Lemma 2 of [9] become (see [9], Section

$$
\begin{aligned}
& \mathcal{W}\left((1+\theta) \tau ; h_{0}\right) \leq(1+K \theta) \mathcal{W}\left(\tau ; h_{0}\right), \\
& \mathcal{W}\left((1-\theta) \tau ; h_{0}\right) \geq(1-K \theta) \mathcal{W}\left(\tau ; h_{0}\right),
\end{aligned}
$$


for a suitable $K>0$. Then, repeating the same arguments as in the proof of Proposition 5 and of Theorem 1, we obtain the following result:

THeOREM 2. Let $p_{0}$ be quasi-elliptic with respect to $\xi$. Let $\mathcal{N}_{\epsilon}(\tau)$ be the counting function associated to the operator $h_{\epsilon}^{w}$. Under the hypotheses of Theorem 1, for

$$
\begin{gathered}
0<d<\frac{4}{3}, \\
0<\mu<\frac{2}{3} \frac{1-\delta m(\mathcal{P})}{m(\mathcal{P})}, \\
\mu \leq(1-\rho)(1-\zeta),
\end{gathered}
$$

with

$$
\zeta=\max _{\alpha \in A \backslash F(\mathcal{P})} k(\alpha ; \mathcal{P})
$$

we have that

$$
\mathcal{N}_{\epsilon}(\tau)=\epsilon^{-2 n} \mathcal{W}\left(\tau ; h_{0}\right)\left\{1+O\left(\epsilon^{d} \tau^{-\mu}\right)\right\}
$$

as $\tau \rightarrow+\infty$ and for all $0<\epsilon \leq 1$.

Remark. From Theorem 2 it is clear that estimates (7),(8) and (9) can be improved with the following ones:

$$
\begin{gathered}
0<d<\frac{4}{3} \\
0<\mu<\frac{2}{3} \frac{1-\delta m(\mathcal{P})}{m(\mathcal{P})}, \\
\mu \leq(1-\rho)(1-\zeta) .
\end{gathered}
$$

Moreover, in the quasi-elliptic case, the Weyl term $\mathcal{W}\left(\tau ; h_{0}\right)$ can be expressed in a more explicit form:

$$
\mathcal{W}\left(\tau ; h_{0}\right)=(2 \pi)^{-n} \int(\tau-q(x))_{+}^{|\omega|} \sigma_{\omega}(x) d x
$$

where $(\tau-q(x))_{+}$is the positive part of $\tau-q(x)$,

$$
\sigma_{\omega}(x)=\frac{1}{|\omega|} \int_{\Psi} p_{0}(x, \zeta(\psi))^{-|\omega|}\left|J_{\omega}(\psi)\right| d \psi,
$$

and $\left|J_{\omega}(\psi)\right|$ is the Jacobian of a suitable matrix (see [9], Section 4, for further details).

\section{REFERENCES}

[1] P. Boggiatto, E. Buzano, and L. Rodino, Global hypoellipticity and spectral theory, Mathematical Research, vol. 92, Akademie Verlag, Berlin, 1996.

[2] N. Dencker, The Weyl calculus with locally temperate metrics and weights, Archiv för Math., 24 (1986), pp. 59-79.

[3] L. HöRMANDER, On the asymptotic distribution of the eigenvalues of pseudodifferential operators in $\mathbf{R}^{n}$, Archiv för Math., 17 (1979), pp. 297-313.

[4] - The Weyl calculus of pseudo-differential operators, Comm. Pure Appl. Math., 32 (1979), pp. 359-443. 
[5] Pham The LaI, Comportament asymptotique des valeurs propres d'une classe d'operateur de type Schrödinger, J. Math. Kyoto Univ., 18:2 (1978), pp. 353-375.

[6] M. A. Shubin, Pseudodifferential operators and spectral theory, second ed., Springer-Verlag, Berlin, 2001.

[7] A. Ziggioto, Comportamento asintotico dello spettro di operatori multi-quasi-ellittici di tipo Schrödinger, Bollettino U.M.I., IV-A:8 (2001), pp. 567-569.

[8] Q Quasiclassical analysis of hypoelliptic operators, Rend. Sem. Mat. Univ. Pol. Torino, 61:1 (2003), pp. 85-99.

[9] A. Ziggioto And E. Buzano, Weyl formula for multi-quasi-elliptic operators of Schrödingertype, Annali di Matematica Pura e Applicata, (2001), no. 180, pp. 223-243.

[10] - Weyl formula for hypoelliptic operators of Schrödinger type, Proc. of AMS, 131:1 (2002), pp. 265-274. 